\title{
Mediating Role of Self-Concordance on the Relationship Between Internet Altruistic Behaviour and Subjective Wellbeing
}

\author{
Xianliang Zheng, Fangwei Xie, and Liang Ding \\ School of Educational Science, Gannan Normal University, Ganzhou, People's Republic of China
}

\begin{abstract}
$\mathrm{T}$ he aim of the present study was to examine the mediating effect of self-concordance on the relationship between internet altruistic behaviour (IAB) and subjective wellbeing (SWB). A total of 356 Chinese university students (159 males and 197 females) participated, using the questionnaires in the Internet Altruistic Behavior Scale (IABS), the Self-Concordance Scale (SCS) and the General Well-Being Schedule (GWBS). Correlation results indicated that IAB was positively correlated with self-concordance and SWB. The hierarchical regression analyses indicated that both IAB and self-concordance positively affect SWB. Moreover, IAB affected SWB indirectly via self-concordance, which confirmed selfconcordance partially mediated the relationship between IAB and SWB. These findings extend the previous research on the association between altruism and SWB.
\end{abstract}

Keywords: internet altruistic behaviour, self-concordance, subjective wellbeing, mediation, university students

During the past several decades, many researchers in the positive psychology field have focused on subjective wellbeing (SWB; e.g., Frederick, Purrington, \& Dunbar, 2016; Newman, Tay, \& Diener, 2014; Ozcakir, Dogan, Cakir, Bayram, \& Bilgel, 2014). SWB refers to people's evaluations of their lives according to the dimensions of life satisfaction, positive affect, and negative affect (Diener, 1994). If a person is satisfied with life, usually feeling positive affect and seldom experiencing negative affect, he or she will enjoy a high level of wellbeing. The research on SWB is of practical significance to improve individuals' life quality. To achieve a deep understanding of wellbeing, researchers have focused on the social and psychological influcences on individuals' wellbeing (e.g., DeNeve \& Cooper, 1998; Moynihan, DeLeire, \& Enami, 2015; Wang, Qi, \& Cui, 2014).

Altruism refers to behaviour that is directly and intentionally aimed at benefitting another person (Pareek \& Jain, 2012), and it is very common in human interactions. In recent years, as the internet has become more popular, altruism has become widespread in cyberspace. Therefore, internet altruistic behaviour (IAB) has attracted the attention of some researchers (e.g., Nicolas, 2008; Wright \& Li, 2011; Zheng \& Zhao, 2015). IAB is defined as behaviour performed voluntarily to help others on the internet, such as sharing successful experiences with others on the internet, recommending good articles, music or films on Facebook or Twitter, or helping others solve their problems online, and so on, when there is no expectation of getting any prizes or rewards and there is no external pressure from others (Zheng \& Zhao, 2015). Altruistic behaviour on the internet is different from altruism occurring in other contexts. Wallace (2001) pointed out that compared to face-to-face situations, people are more willing to help others on the internet. In contrast with the physical world, the advantages of the internet are that it is more conducive to the occurrence of altruistic behaviour in cyberspace (Zheng, 2010). For example, the anonymity of the internet promotes people's self-disclosure, which is easier for their help-seeking behaviour and more likely to get others' attention or sympathy on the internet. And the timeliness and interactivity of the internet may enable higher efficiency and a lower cost for altruistic behaviour.

In the available literature, plenty of researches have been conducted to explore the relationship between altruism and SWB (e.g., Moynihan et al., 2015; Müller, Ziegelmann, Simonson, Tesch-Römer, \& Huxhold, 2014; Pareek \& Jain, 2012), but little research has elaborated the

This research was supported by the National Natural Science Foundation of China (31760286).

Address for correspondence: Xianliang Zheng, School of Educational Science, Gannan Normal University, Rongjiang New District, Ganzhou 341000 , People's Republic of China. Email: zxl2004@sina.com 
potential mechanism underlying the relationship between internet altruism and SWB. Is there a possibility that some of the advantages of internet altruism (e.g., anonymity, timeliness, and interactivity) would make a difference in the relationship between altruistic behaviours and SWB? Would people's altruism in the internet context promote or reduce their SWB? And how could individuals' internet altruism affect their SWB? This article aims to examine the main effect of IAB on SWB, as well as the mediation of self-concordance for this relationship in the Chinese culture context.

\section{Internet Altruistic Behaviour and Subjective Wellbeing}

Numerous researchers have investigated the relationship between altruism and SWB, and there is ample evidence that SWB is linked to individuals' altruism. For instance, Moynihan et al. (2015) pointed out that altruism improved SWB by replacing more negative mood, because it becomes more difficult to indulge in a negative mood like sadness, anger, or fear if a person is focused on offering help. Findings have shown that altruism can improve social relationships, raise gratification, and reduce negative emotions, and hence promote individuals' wellbeing (Bartlett \& DeSteno, 2006; Glomb, Bhave, Miner, \& Wall, 2011; Grant \& Gino, 2010). Tian, Du, and Huebner (2015) revealed that altruistic behaviour was positively correlated with life satisfaction and positive affect. Findings reported by several empirical studies have also shown that altruistic behaviour leads to a high level of SWB and predicts SWB (Aknin, Hamlin, \& Dunn, 2012; Oarga, Stavrova, \& Fetchenhauer, 2015; Pareek \& Jain, 2012).

$I A B$ is a new form of altruism in the internet era, which is the extension into cyberspace of altruistic behaviour in the physical world. As a result of the differences of the context between the internet and physical world, IAB may differ from traditional forms of altruism that have been studied (e.g., picking up dropped books for a stranger). For example, on the internet, people's altruistic behaviour may be more likely to lead to becoming socially approved (e.g., reciprocal altruism). When people engage in more $\mathrm{IAB}$, they may be experiencing collateral social acceptance (or perceived social acceptance). Researchers, however, have pointed out that IAB and physical-world altruistic behaviour are similar in nature as they are both aimed at benefitting another person (Zheng, 2010). The coconstruction theory (Subrahmanyam \& Greenfield, 2008) posits that individuals are psychologically connected to their online worlds similarly to their offline worlds. People may produce positive emotions, such as enjoyment and pleasure, and intrinsic rewards after helping others both in face-to-face situations and an internet context. Empirical studies also showed that face-to-face altruistic behaviour was positively associated with engagement in IAB (Wright \& Li, 2011; Zheng, 2010, 2013). As mentioned above, faceto-face altruistic behaviour was thought to have close links with SWB. So, we proposed that IAB was in close relations to SWB. In other words, IAB would promote individual's SWB.

\section{Self-Concordance and Subjective Wellbeing}

Self-concordance is defined as the extent to which a goal is in accordance with a person's underlying interests, feelings, desires, and values, relative to being caused by external reasons (Sheldon \& Elliot, 1999). According to self-concordance theory (Sheldon \& Elliot, 1999), and as also indicated by some researchers in the literature, self-concordance has been positively associated with SWB. For instance, researchers have shown that SWB will tend to be enhanced when individuals' behaviours fulfill their essential, inherent needs, and these are selfdetermined, rather than commanded or forced by external pressure (Kelly, Mansell, \& Wood, 2015; Niemiec, Ryan, \& Deci, 2009; Smith, Ntoumanis, Duda, \& Vansteenkiste, 2011). Sheldon and Houser-Marko (2001) showed that self-concordance predicted longitudinal increases in SWB by way of the greater goal attainment inspired by selfconcordance. Tadić, Bakker, and Oerlemans (2013) reported that teachers with high self-concordance have greater SWB than teachers with low self-concordance. Sheldon et al. (2004) also assessed the relationship between self-concordance and SWB in four different cultures (U.S., Chinese, South Korean, and Taiwanese cultures), and the results showed that self-concordance was significantly positively correlated with life-satisfaction, positive affect, and aggregate SWB. Self-concordance predicted SWB within every culture. Therefore, as seen from these empirical studies, self-concordance does play a vital role in the promotion of SWB.

\section{Internet Altruistic Behaviour and Self-Concordance}

Self-concordance theory, as proposed by Sheldon and Elliot (1999), argues that individuals derive satisfaction as they pursue goals that align with their values and interests, especially goals that are autonomously selected rather than externally imposed. According to self-concordance theory, when individuals believe goals that they are pursuing are only to gain extrinsic prizes or to fulfill duties imposed upon them, their self-concordance will be low. In contrast, when people perceive goals they are pursuing reflect their interests, desires and values, or when they find the goals intrinsically interesting and enjoyable, their self-concordance will be high. People perform IAB in line with their own interests, desires and values, which is intrinsically motivated, rather than driven by external pressure. After helping others in cyberspace, individuals may feel elated and joyous, and it may also produce intrinsic rewards - for example, feelings of satisfaction or accomplishment. Moreover, compared with physical-world altruism, IABs are less likely to be imposed by others; and individuals may have more opportunities to follow the progress of their goals regarding altruism on the internet. 
Hence, we believed that IAB would be conducive to the improvement of self-concordance.

\section{Current Study}

Although many researchers have provided evidence for the positive relationship between physical-world altruistic behaviour and SWB, few studies have investigated how IAB affects SWB, and what the mechanisms are that can explain this influence. The first aim of the present study was to expand the previous research findings by examining the relationship between IAB and SWB. Additionally, in order to identify the potential mechanism underlying the relationship between IAB and SWB, the study also aimed to examine the mediating effects of self-concordance on the link between IAB and SWB using hierarchical regression analyses. The samples were from a group of Chinese university students. In line with the aforementioned studies, this article proposes the following hypotheses: (1) IAB is positively correlated with self-concordance and SWB; (2) self-concordance is positively correlated with SWB; (3) self-concordance mediates the association between IAB and SWB.

\section{Method}

\section{Participants and Procedure}

A total of 356 university students were randomly drawn from a Chinese university as volunteers to take part in the study. In the sample, $159(44.66 \%)$ were males and $197(55.34 \%)$ were females. Among them, a total of 82 (23.03\%) were freshmen, 107 (30.06\%) were sophomores, 96 (26.97\%) were juniors, and 71 (19.94\%) were seniors. The participants were aged from 17-24 years old with a mean age of 20.64 years $(S D=1.31$ ). Self-report questionnaires were completed by participants in the classroom environment after obtaining informed consent. It took approximately 15 minutes for the participants to complete all the questionnaires.

\section{Measures}

Internet altruistic behaviour. IAB was assessed by using the 26-item Internet Altruistic Behavior Scale (IABS; Zheng, Zhu, \& Gu, 2011). The IABS consists of four subscales: internet-support (nine items), internet-guidance (six items), internet-sharing (six items), and internetreminding (five items). The participants responded on a 4-point Likert scale ranging from 1 (never or very rarely) to 4 (always or very often). It contains items such as, 'Answer some questions online' and 'Bless others on the internet'. Higher scores indicate higher levels of IAB. Many studies have supported that the IABS has good reliability and validity in Chinese populations (e.g., Zheng \& Zhao, 2015). The Cronbach alpha coefficient was .92 in the current study.

Self-concordance. Sheldon and Elliot's (1999) SelfConcordance Scale (SCS) was adapted to assess the participants' self-concordance. First, participants generated two
IABs that had been accomplished during the last month. Second, participants were asked to complete four items developed by Sheldon and Elliot (1999) to assess the motivation for pursuing each of the two IABs (eight items in total). The scale assessed four aspects of motivation: (a) intrinsic (e.g., You pursue the behaviour because of the fun and pleasure it gives); (b) identified (e.g., You pursue the behaviour because you are convinced of its importance); (c) external (e.g., You pursue the behaviour because somebody else or the situation demands it) and (d) introjected (e.g., You pursue the behaviour because you would feel ashamed, guilty, or anxious if you did not). The items were rated on a 7-point Likert scale ranging from 1 (not at all) to 7 (completely). A composite score of self-concordance was computed by summing the intrinsic and identified ratings and then subtracting the external and introjected ratings. Higher scores indicate greater self-concordance. In this study, the Cronbach alpha coefficient was .77.

Subjective wellbeing. SWB was measured with the 18item General Well-Being Schedule (GWBS; McDowell, Newell, \& McDowell, 2006). The GWBS is a widely used self-reported questionnaire measuring SWB. It consists of 14 items that are responded to on a 6-point Likert scale and 4 items that are responded on a 10-point Likert scale. It contains items such as, 'Have you felt anxious or worried during the last month?' ( $1=$ extremely so, $6=$ not at all $)$ and 'During the last month, how depressed or cheerful have you been? ' $(1=$ very depressed, $10=$ very cheerful $)$. Higher scores imply higher levels of SWB. The Chinese version of this questionnaire has satisfactory validity and reliability (e.g., Ma \& Zeng, 2015). The Cronbach alpha coefficient was .84 in the present study.

\section{Data Analysis}

First of all, common method bias was checked with Harman's single-factor test using exploratory factor analysis. Second, the descriptive statistics and the bivariate correlations were used for all the participants to quantitate the relationships among all the study variables. Third, hierarchical regression analyses were performed to test the mediating role of self-concordance in explaining the relation between IAB and SWB. The mediation analysis followed the recommendation of Wen, Chang, Hau, and Liu (2004). As previous studies have shown that age and gender are correlated with SWB (Huo \& Kong, 2014; Tu \& Zhang, 2015), we controlled for age and gender in the hierarchical regression analyses. Age was a numerical value, and gender was defined as discrete, with 0 for males, and 1 for females. Lastly, the bootstrap procedure recommended by Preacher, Rucker, and Hayes (2007) were used to test the statistical significance of the indirect effect for IAB on SWB. All the statistical analyses (i.e., descriptive statistics, correlations, hierarchical regressions) were performed with the statistical software package SPSS 16.0. The criterion of $p<.05$ was set to define statistical significance. 


Table 1
\begin{tabular}{lrrrrrrr} 
Descriptives and Correlations & Among the Study Variables \\
\hline Variables & $M$ & SD & Skewness & Kurtosis & IAB & Self-concordance & SWB \\
\hline IAB & 52.76 & 13.58 & .48 & .40 & 1 & & \\
Self-concordance & 3.45 & 2.69 & .27 & -.37 & $.33^{* *}$ & 1 & \\
SWB & 76.32 & 10.46 & -.42 & .80 & $.29^{* *}$ & $.26^{* *}$ & 1 \\
\hline
\end{tabular}

Note: $\mathrm{IAB}=$ internet altruistic behaviour, SWB = subjective well-being.

$* * p<.01$.

\section{Results}

\section{Common Method Bias Assessing}

All the data in this study were collected from the selfreported questionnaires, which are subject to the risk of common method bias (Podsakoff, MacKenzie, Lee, \& Podsakoff, 2003). We therefore performed Harman's singlefactor test, which has been widely used in previous research to check the common method bias (e.g., Aulakh, \& Gencturk, 2000; Karatepe, 2011; Krishnaveni \& Deepa, 2013). The exploratory factor analysis was applied for Harman's single-factor test in this study. We selected all the study variables into exploratory factor analysis, using unrotated principal component factor analysis to determine the number of factors that are essential to explain the variance in the study variables. The results of the factor analysis showed that there were 14 distinct factors with eigenvalues bigger than 1.0. The 14 factors accounted for $64.66 \%$ of the total variance, and the first largest factor accounted for only $20.25 \%$ of the variance, less than $40 \%$. The results thus indicated that common method bias was not a significant problem for this study.

\section{Preliminary Analyses}

Table 1 presents the descriptive statistics for the variables of IAB, self-concordance, and SWB, and the correlations among these measures. Skewness and kurtosis values showed that all the study variables were in normal distribution. The bivariate correlations indicated that IAB was positively correlated with self-concordance $(p<.01)$ and SWB $(p<.01)$ for university students; namely, the higher the level of IAB, the greater was their self-concordance and SWB. Likewise, self-concordance was positively related with SWB $(p<.01)$ for university students, that is, the stronger the self-concordance, the higher the SWB.

\section{Mediation Analyses}

In order to test whether self-concordance mediates the relationship between IAB and SWB, hierarchical regression analyses were performed by employing the procedures described by Wen et al. (2004). In this study, the following three regression equations were evaluated: (a) the dependent variable (SWB) was regressed on the independent variable (IAB); (b) the mediator (self-concordance) was regressed on the independent variable (IAB); and (c) the dependent variable (SWB) was regressed on both the mediator (self-concordance) and the independent variable

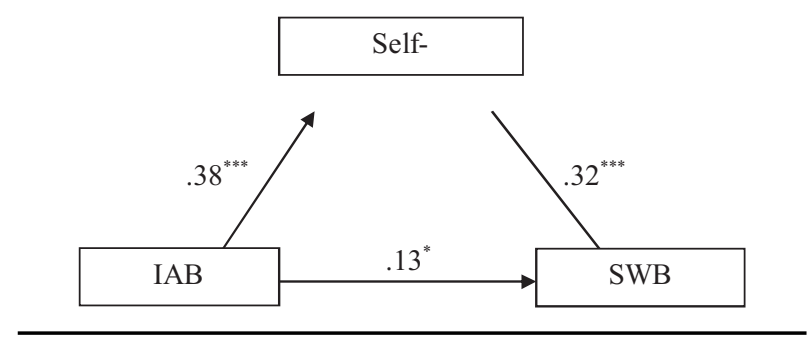

Figure 1

Beta coefficients for the mediating effect of self-concordance between internet altruistic behaviour and subjective wellbeing.

Note: ${ }^{*} p<.05,{ }^{* * *} p<.001$. SWB $=$ subjective wellbeing, IAB $=$ internet altruistic behaviour.

(IAB). Each regression equation analysis was controlled by age and gender.

Table 2 presents the results of the mediation analyses. In regression equation 1 , IAB significantly predicted SWB. In regression equation 2 , IAB significantly predicted self-concordance. In equation 3 , after controlling for age, gender, and self-concordance, the independent effect of IAB on SWB decreased, but IAB continued to significantly predict SWB. Hence, the relationship between IAB and SWB is partially mediated by self-concordance, and the size of the mediating effect was $.38 \times .32=.12$, which accounted for $.12 /(.12+.13)=48 \%$ of all the effects. Figure 1 displays the the mediation model we established.

Following procedures described by Preacher et al. (2007), we moved forward to use the bias-corrected bootstrapping method to construct the $95 \%$ confidence interval for the indirect effect. We created 5,000 bootstrap samples $(N=356)$ from the original data-set by random sampling. The results from 5,000 bootstrap samples indicated that the $95 \%$ confidence interval for the indirect effect ranged from .08 to .26 and did not include zero. We can conclude that the indirect effect is significantly different from zero at $\alpha=.05$. Thus, the results provide clear support for hypothesis 3 , which proposed that selfconcordance would mediate the relationship between IAB and SWB.

\section{Discussion}

Altruism is behaviour that is aimed at benefitting another person. Altruism has been shown to be associated with individuals' wellbeing (Moynihan et al., 2015; Pareek \& Jain, 2012; Tian, et al., 2015), but researchers have predominantly focused on how physical-world altruism increases 


\begin{tabular}{|c|c|c|c|c|c|c|c|c|}
\hline Regression equation & Dependent variable & Independent variable & B & $S E$ & $\beta$ & $r^{2}$ & $\Delta r^{2}$ & $\Delta F$ \\
\hline \multirow[t]{3}{*}{1} & \multirow[t]{3}{*}{ SWB } & Age & -1.19 & .47 & $-.16^{*}$ & .08 & .04 & $9.96 * *$ \\
\hline & & Gender & .73 & .34 & $.15^{*}$ & & & \\
\hline & & $I A B$ & .32 & .11 & $.25^{* *}$ & & & \\
\hline \multirow[t]{3}{*}{2} & \multirow[t]{3}{*}{ Self-concordance } & Age & .08 & .12 & .04 & .11 & .06 & $13.75^{* * *}$ \\
\hline & & Gender & 1.48 & .53 & $.18^{*}$ & & & \\
\hline & & $I A B$ & .44 & .08 & $.38^{* * *}$ & & & \\
\hline \multirow[t]{4}{*}{3} & \multirow[t]{4}{*}{ SWB } & Age & -1.22 & .47 & $-.17^{* *}$ & .12 & .08 & $15.36^{* * *}$ \\
\hline & & Gender & .69 & .39 & .09 & & & \\
\hline & & Self-concordance & .57 & .12 & $.32 * * *$ & & & \\
\hline & & IAB & .10 & .05 & $.13^{*}$ & & & \\
\hline
\end{tabular}

Note: $\mathrm{SWB}=$ subjective wellbeing, $\mathrm{IAB}=$ internet altruistic behaviour.

$* p<.05,{ }^{* *} p<.01,{ }^{* * *} p<.001$

individuals' wellbeing. Currently, with the rapid advances in technology and the popularity of the internet, altruism on the internet has become widespread and it has become an important form of altruism. Owing to the uniqueness of the internet context (e.g., anonymity, timeliness, and interactivity), IAB is somewhat different from face-to-face altruistic behaviour (Zheng, 2010). However, the association between IAB and wellbeing has not yet been investigated in the literature. The main aim of this article was to examine the mediating role of self-concordance on the relationship between IAB and SWB in a sample of Chinese university students. Consistent with our hypotheses, correlational results showed that IAB was positively correlated with SWB. Those university students who perform more altruistic behaviour in cyberspace are more likely to have greater SWB. This suggests that IAB is a significant predictor for SWB. We argue that university students who engage in IAB do not expect any external prizes, but they may gain some internal rewards such as feelings of accomplishment, satisfaction or enjoyment after offering help, and simultaneously, the recipients can also give them affirmation, encouragement, or praise for their altruistic behaviour on the internet. Consequently, their SWB will be promoted. Our findings indicate that altruistic behaviour can improve SWB not only in face-to-face situations but also in cyberspace, which expands upon previous studies on the association between altruism and SWB (James, 2011; Oarga el al., 2015; Stavrova, Fetchenhauer, \& Schlösser, 2013). Furthermore, the results of bivariate correlations suggest that self-concordance is positively correlated with SWB, which is in line with the results from previous research on the relation between self-concordance and SWB (Chirkov, Ryan, Kim, \& Kaplan, 2003; Kelly et al., 2015; Koletzko, Herrmann, \& Brandstätter, 2015).

As expected, the results of our study confirmed that IAB and SWB were mediated by self-concordance. Selfconcordance acted as a partial mediator of the relationship between IAB and SWB. The results indicated that individuals with greater IAB are not only predisposed to improve their SWB, but also have higher levels of selfconcordance, which in turn contributed to an increase in their SWB. The current results are consistent with the self-concordance theory proposed by Sheldon and Elliot (1999), in which it is suggested that individuals who carry out behaviours that are in line with their interests and values will obtain greater SWB. IAB is aimed at benefitting another person in cyberspace. When individuals perform altruistic behaviour in cyberspace, they will perceive that they are pursuing goals reflecting their true interests and values, or they will find their altruistic behaviours intrinsically enjoyable and interesting, which will enhance their SWB. In the meantime, individuals' self-concordance will be high, and they will experience the benefit of increased SWB. That is, SWB is not only directly influenced by IAB, but also indirectly through self-concordance. Moreover, our results showed that the size of the mediating effect of self-concordance only accounted for $48 \%$ of all the effects, which indicated that in addition to self-concordance, some other factors (e.g., personality traits and social support) probably also mediate the effect of IAB on SWB. Our further exploration will focus on this area.

There were several limitations of the present study. First, the participants in our study were relatively homogenous as they were all university students from China, which limited the generalisability of our findings to other populations and cultures. Hence, in the future, it would be useful to reexamine the results in different populations to generalise the present findings. Second, our study used a cross-sectional design, but the evidence offered by the cross-sectional study was not strong enough to confirm the causal relationship between IAB, self-concordance, and SWB. Therefore, experimental designed studies are needed to shed light on this issue in future studies. Third, all the variables in our study were measured based on selfreport data, which are susceptible to common method bias. Although the results of Harman's single-factor test by using exploratory factor analysis showed that common method basis was not serious in the current study, it would be beneficial to collect data from multiple sources in future research.

Despite these limitations, the present study is the first attempt to explore the relationship between IAB, selfconcordance, and SWB in a sample of Chinese university students. Our findings provide empirical evidence for 
the hypothesis that self-concordance mediates the positive relationship between IAB and SWB, which makes a contribution to the increasing literature of positive psychology. The results of the present study also provide useful suggestions for potential interventions aimed at improving individuals' wellbeing. For example, as IAB is a positive predictor of SWB, it is appropriate that students are encouraged and guided to perform more IAB to enhance their wellbeing. Additionally, the present study highlights the importance of self-concordance in the relationship between IAB and SWB. A high level of self-concordance can help students to increase their SWB. Self-concordance should be underscored when considering the influence of IAB on SWB. It is imperative that teachers and other educators take appropriate steps to enhance students' selfconcordance.

\section{References}

Aknin, L.B., Hamlin, J.K., \& Dunn, E.W. (2012). Giving leads to happiness in young children. Plos One, 7, 1-4.

Aulakh, P.S., \& Gencturk, E.F. (2000). International principalagent relationships: Control, governance and performance. Industrial Marketing Management, 29, 521-538.

Bartlett, M.Y., \& DeSteno, D. (2006). Gratitude and prosocial behavior helping when it costs you. Psychological Science, 17, 319-325.

Chirkov, V., Ryan, R.M., Kim, Y., \& Kaplan, U. (2003). Differentiating autonomy from individualism and independence: $\mathrm{A}$ self-determination theory perspective on internalization of cultural orientations and well-being. Journal of Personality and Social Psychology, 84, 97-109.

DeNeve, K.M., \& Cooper, H. (1998). The happy personality: A meta-analysis of 137 personality traits and subjective wellbeing. Psychological Bulletin, 124, 197-229.

Diener, E. (1994). Assessing subjective well-being: Progress and opportunities. Social Indicators Research, 31, 103-157.

Frederick, T., Purrington, S., \& Dunbar, S. (2016). Differentiation of self, religious coping, and subjective well-being. Mental Health, Religion \& Culture, 19, 553-564.

Glomb, T.M., Bhave, D.P., Miner, A.G., \& Wall, M. (2011). Doing good, feeling good: Examining the role of organizational citizenship behavior in changing mood. Personnel Psychology, 64, 191-223.

Grant, A.M., \& Gino, F. (2010). A little thanks goes a long way: Explaining why gratitude expressions motivate prosocial behavior. Journal of Personality and Social Psychology, 98, 946955.

Huo, Y., \& Kong, F. (2014). Moderating effects of gender and loneliness on the relationship between self-esteem and life satisfaction in Chinese university students. Social Indicators Research, 118, 305-314.

James, H.S. (2011). Is the just man a happy man? An empirical study of the relationship between ethics and subjective wellbeing. Kyklos, 64, 193-212.

Karatepe, O.M. (2011). Do job resources moderate the effect of emotional dissonance on burnout? A study in the city of Ankara, Turkey. International Journal of Contemporary Hospitality Management, 23, 44-65.

Kelly, R.E., Mansell, W., \& Wood, A.M. (2015). Goal conflict and well-being: A review and hierarchical model of goal conflict, ambivalence, self-discrepancy and self-concordance. Personality and Individual Differences, 85, 212-229.

Koletzko, S.H., Herrmann, M., \& Brandstätter, V. (2015). Unconflicted goal striving: Goal ambivalence as a mediator between goal self-concordance and well-being. Personality and Social Psychology Bulletin, 41, 140-156.

Krishnaveni, R., \& Deepa, R. (2013). Controlling common method variance while measuring the impact of emotional intelligence on well-being. Vikalpa: The Journal for Decision Makers, 38, 41-47.

Ma, Z.W., \& Zeng, W.N. (2015). Gender differences in Chinese adolescents' subjective well-being: The mediating role of self-efficacy. Psychological Reports: Sociocultural Issues in Psychology, 116, 311-321.

McDowell, I., Newell, C., \& McDowell, I. (2006). Measuring health: A guide to rating scales and questionnaires. Vol. 268. New York: Oxford University Press.

Moynihan, D.P., DeLeire, T., \& Enami, K. (2015). A life worth living: Evidence on the relationship between prosocial values and happiness. American Review of Public Administration, 45, 311-326.

Müller, D., Ziegelmann, J.P., Simonson, J., Tesch-Römer, C., \& Huxhold, O. (2014). Volunteering and subjective well-being in later adulthood: Is self-efficacy the key? International Journal of Developmental Science, 8, 125-135.

Newman, D.B., Tay, L., \& Diener, E. (2014) Leisure and subjective well-being: A model of psychological mechanisms as mediating factors. Journal of Happiness Studies, 15, 555-578.

Nicolas, G. (2008). Helping on the web: Ethnic stereotypes and computer-mediated communication. Research Journal of Social Sciences, 3, 1-3.

Niemiec, C.P., Ryan, R.M., \& Deci, E.L. (2009). The path taken: Consequences of attaining intrinsic and extrinsic aspirations in post-college life. Journal of Research in Personality, 43, 291306.

Oarga, C., Stavrova, O., \& Fetchenhauer, D. (2015). When and why is helping others good for well-being? The role of belief in reciprocity and conformity to society's expectations. European Journal of Social Psychology, 45, 242-254.

Ozcakir, A., Dogan, F.O., Cakir, Y.T., Bayram, N., \& Bilgel, N. (2014). Subjective well-being among primary health care patients. Plos One, 9, 1-15.

Pareek, S., \& Jain, M. (2012). Subjective well-being in relation to altruism and forgiveness among school going adolescents. International Journal of Psychology and Behavioral Sciences, 2, 138-141.

Podsakoff, P.M., MacKenzie, S.M., Lee, J., \& Podsakoff, N.P. (2003). Common method variance in behavioral research: A critical review of the literature and recommended remedies. Journal of Applied Psychology, 88, 879-903.

Preacher, K.J., Rucker, D.D., \& Hayes, A.F. (2007). Addressing moderated mediation hypotheses: Theory, methods, and prescriptions. Multivariate Behavioral Research, 42, 185-227. 
Sheldon, K.M., \& Elliot, A.J. (1999). Goal striving, need satisfaction, and longitudinal well-being: The self-concordance model. Journal of Personality and Social Psychology, 76, 546557.

Sheldon, K.M., Elliot, A.J., Ryan, R.M., Chirkov, V., Kim, Y., Wu, C., ... Sun, K. (2004). Self-concordance and subjective wellbeing in four cultures. Journal of Cross-Cultural Psychology, 35, 209-223.

Sheldon, K.M., \& Houser-Marko, L., (2001). Self-concordance, goal attainment, and the pursuit of happiness: can there be an upward spiral? Journal of Personality \& Social Psychology, 80, 152-165.

Smith, A.L., Ntoumanis, N., Duda, J.L., \& Vansteenkiste, M. (2011). Goal striving, coping, and well-being: A prospective investigation of the self-concordance model in sport. Journal of Sport \& Exercise Psychology, 33, 124-145.

Stavrova, O., Fetchenhauer, D., \& Schlösser, T. (2013). Why are religious people happy? The effect of the social norm of religiosity across countries. Social Science Research, 42, 90105.

Subrahmanyam, K., \& Greenfield, P. (2008). Communicating online: Adolescent relationships and the media. The Future of Children: Children and Media Technology, 18, 119-146.

Tadić, M., Bakker, A.B., \& Oerlemans, W.G.M. (2013). Work happiness among teachers: A day reconstruction study on the role of self-concordance. Journal of School Psychology, 51, $735-750$.

Tian, L.L., Du, M.M., \& Huebner, E.S. (2015).The effect of gratitude on elementary school students' subjective well-being in schools: the mediating role of prosocial behavior. Social Indicators Research, 122, 87-904.
Tu, Y.D., \& Zhang, S.X. (2015). Loneliness and subjective wellbeing among Chinese undergraduates: The mediating role of self-efficacy. Social Indicators Research, 124, 963-980.

Wallace, P.M. (2001). The psychology of the internet. Cambridge: Cambridge University Press.

Wang, J., Qi, L., \& Cui, L.J. (2014). The mediating effect of personality traits on the relationship between self-concealment and subjective well-being. Social Behavior and Personality, 42, 695-703.

Wen, Z.L., Chang, L., Hau, K.T., \& Liu, H.Y. (2004). Testing and application of the mediating effects [In Chinese]. Acta Psychologica Sinica, 36, 614-620.

Wright, M.F., \& Li, Y. (2011). The associations between young adults' face-to-face prosocial behaviors and their online prosocial behaviors. Computers in Human Behavior, 27, 1959-1962.

Zheng, X.L. (2010). Internet altruistic behavior of undergraduates: Scale development and multilevel analysis [In Chinese]. Unpublished doctoral dissertation, Shanghai Normal University.

Zheng, X. (2013). The relationship between altruistic behavior in the real world and Internet altruistic behavior: The role of online social support [In Chinese]. Psychological Development and Education, 21, 31-37.

Zheng, X.L., \& Zhao, W. (2015). Relationship between internet altruistic behavior and hope of middle school students: The mediating role of self-efficacy and self-esteem [In Chinese]. Psychological Development and Education, 31, 428-436.

Zheng, X.L., Zhu, C.L., \& Gu, H.G. (2011). Development of internet altruistic behavior scale for college students [In Chinese]. Chinese Journal of Clinical Psychology, 19, 606-608. 